\title{
Maternal and perinatal outcomes: Preterm premature rupture of membrane between 34 - 37 weeks
}

\author{
Authors \\ Dr Ramakanta Sahoo ${ }^{1 *}$, Prof (Dr) Pratima Mishra ${ }^{2}$ \\ Department of Obstetrics and Gynecology, Hi-Tech Medical College \& Hospital, Bhubaneswar, \\ Odisha, India \\ *Corresponding Author \\ Dr Ramakanta Sahoo \\ $3^{\text {rd }}$ yr PG Student, Dept. of Obstetrics \& Gynaecology, Hi-Tech Medical College \& Hospital, \\ Bhubaneswar, 751010 , Odisha, India \\ Email: dr.rksahoo1234@gmail.com
}

\begin{abstract}
Background: This is an Prospective observational analytical study carried out in department of obstetrics and Gynecology, Hi-Tech Medical College \& Hospital, Bhubaneswar, in a tertiary care center to determine the factors influencing fetal and maternal outcome, prognosis, morbidity and mortality in preterm premature rupture of membrane cases.

Methods: The present study is a prospective observational study of perinatal and maternal outcome in 100 cases of preterm premature rupture of membranes in between 34-37 weeks gestation with singleton pregnancy, from 1st Nov 2016 to $31^{\text {st }}$ Oct 2018. Patients with medical complications like anemia, preexisting hypertension, diabetes, vascular or renal disease, multiple gestations, uterine or fetal anomalies etc. are excluded from the study. Detailed history, physical examinations were carried out and appropriate management instituted as per individual patients need.

Results: In this study maternal morbidity was 35\%. Perinatal morbidity was $68 \%$ and most common causes were hyperbilirubinemia (23\%), Sepsis (15\%). Perinatal mortality was seen in $1 \%$ and mainly due to very LBW. Fifty Eight (58\%) neonates were delivered by cesarean. The main indications for cesarean being fetal distress (43.3\%) followed by non progress of labour (36\%).

Conclusions: PPROM is one of the important causes of preterm birth that can result in high perinatal morbidity and mortality along with maternal morbidity. Looking after a premature infant puts immense burden on the family, economy and health care resources of the country. An understanding of gestational age dependent neonatal morbidity and mortality is important in determining the potential benefits of conservative management of preterm PROM at any gestation.

Keywords: PPROM, Perinatal morbidity.
\end{abstract}

\section{Introduction}

Premature rupture of membranes (PROM) is defined as the spontaneous rupture of amniotic membrane with a release of amniotic fluid at least one hour before the onset of labor. If the membranes rupture after 37 weeks of gestation it is called term PROM. If the rupture of membranes (ROM) occur after 28 weeks but 
before 37 weeks of gestation is termed as the preterm premature rupture of membrane (PPROM). ${ }^{1}$

Latent period: It is the time interval between the rupture of membranes and the onset of uterine contractions. $^{2}$

Prolonged PROM: It is the term used when more than 24 hours have elapsed before the labor ensues. $^{2}$

High rupture of Membranes- It is due to the rupture of amniochorion at a site distant from internal OS and spontaneous cessation of leakage can occur. ${ }^{2}$

PROM is usually followed by labor. The onset of labor after PROM is directly related to the gestational age at the time of rupture. Labor started within 24 hours of PROM in $81 \%$ of patients carrying babies larger than 2500 grams.

Only $48 \%$ of the patients develop labor within three days of PROM. $^{2}$ It is an obstetric conundrum which is poorly defined, with an obscure etiology, difficult to diagnose and is associated with significant maternal and neonatal morbidity and mortality and has diverse and controversial management strategies. 2

\section{Incidence of Prom}

PROM occurs in approximately $10 \%$ of all pregnancies and in $70 \%$ of the cases at term. Although there is some morbidity when PROM occurs in term pregnancies, the fundamental clinical problem is preterm PROM, a condition that occurs in $3 \%$ of all pregnancies and is responsible for approximately $30 \%$ of all preterm deliveries. $^{1}$

Preterm PROM complicates 3-8\% of pregnancies and leads to one third of preterm deliveries.2 It increases the risk of prematurity and leads to other perinatal and neonatal complications with 1$2 \%$ risk of fetal death. PROM is associated with increased risk of chorioamnionitis, dysfunctional labor, increased cesarean rates, postpartum hemorrhage and endometritis in the mother. In the fetus, there is increased occurrence of hyaline membrane disease, intraventricular hemorrhage, sepsis, cord prolapse, fetal distress and increased fetal wastage.

Thus, earlier the gestational age at the time of PROM, longer is the latency and more the complications. Management of PROM remains controversial and challenging. ${ }^{2}$ Controversy surrounds the role of tocolytics, steroids and antibiotics. $^{3}$

The aim of the study was to observe the maternal and perinatal outcome in patients with preterm premature rupture of membranes; to study the maternal complications in preterm premature rupture of membranes; to find out the perinatal morbidity and mortality in preterm premature rupture of membranes and to study mode of delivery in preterm premature rupture of membranes.

\section{Material and Methods}

Source of data: 100 patients of preterm premature rupture of membranes in between 34 37 weeks gestation admitted in labor room were studied after considering inclusion and exclusion criteria.

Study design: Hospital based prospective observational study.

Study period: Over a period of two year from Nov 2016 to October2018.

Study place: Tertiary care

hospital. Sample size: 100.

As per statistical formula

sample size was 100 .

\section{Inclusion criteria}

- All pregnant women with a singleton pregnancy between 34-37 weeks of gestational age with preterm premature rupture of membranes.

\section{Exclusion criteria}

- Multiple pregnancies

- Intrauterine growth restriction

- Uterine anomalies

- Fetal anomalies

- Myomauteri

- Hypertensive disorders and pregnancy 
induced hypertension

- Gestational diabetes mellitus

- Antepartum hemorrhage

- Chronic renalfailure

- Class II to IV cardiac diseases

\section{Method of collection of data}

A detailed history was taken including age, booking, socio-economic status, time of onset of leaking, amount of fluid lost, its colour, odour, association with pain or bleeding per vagina and perception of fetal movements.

General examination, height and weight were recorded. Systemic examination included cardiovascular, respiratory systems and CNS systems.

In the obstetric examination, following were noted. Height of uterine fundus, lie, presentation and position of fetus, engagement of presenting part, condition of uterus whether contracted or relaxed.

Uterine tenderness was looked for as a sign of chorioamnionitis. Fetal heart sound was auscultated and its rate, rhythm and tone were noted. A sterile speculum examination was done and amniotic fluid pooling in posterior fornix was observed. The colour and smell of fluid was noted. If no fluid was seen, the patient was asked to cough, and drainage of fluid was looked for. In doubt, vaginal fluid specimen was collected and subjected to litmus paper test. Cervical swab was taken and sent for Gram stain and culture sensitivity.

A single pelvic examination was done to note the Bishop's score, adequacy of pelvis, assessment of CPD and to rule out cord prolapse. Investigations like total count, differential count and C-reactive protein were done. Prophylactic antibiotic in the form of injection ampicillin $1 \mathrm{gm}$ IV every 6 hourly was given.

Depending upon the gestation age and Bishop's score labour was induced with prostaglandins or augmented with oxytocin. Time of induction was noted. Progress of labor was monitored, Induction to delivery interval and PROM to delivery interval were noted. Maternal pulse, blood pressure, fetal heart rate and its variations were checked frequently.

The onset of any complications like fetal distress, fetal heart rate variations, chorioamnionitis (clinical) were looked for. Progress of labour was monitored. If there was any evidence of fetal jeopardy or any other obstetrical complications, labour was cut short by instrumental delivery or cesarean section as required.

Following facts were noted:

- Soon after delivery, APGAR score at 1- and 5- minutes birth weight, sex, congenital anomalies, immediate complications and birth injuries, signs of asphyxia, meconium aspiration, sepsis and other associated complications were recorded.

- The babies were followed up in the postnatal period. Neonatal morbidity and mortality were noted.

- Mothers were watched for third stage complications like $\mathrm{PPH}$ and retained placenta.

They were followed up in puerperal period. Vital parameters like temperature, pulse, blood pressure were frequently monitored. Women were specifically asked for foul smelling lochia and the presence of febrile morbidity. Episiotomy wound, and cesarean section wound was observed, and regular Follow-up was done. Maternal morbidity like puerperal sepsis, urinary and respiratory tract infection and wound infection were looked for.

Both mother and the baby were followed up till their stay in the hospital.

\section{Statistical analysis}

All relevant data will be compiled and entered into computer using computer-based software SPSS for appropriate analysis. Quantitative data will be analyzed by proportion and Chi square test at $p<0.05$ level of significance. 


\section{Results}

It is observed that as the duration of PPROM increases the maternal morbidity also increases. In this study $77.17 \%$ patients had maternal morbidity when duration of PROM exceeded 24hours

Table 1: Duration of PPROM and maternal morbidity

\begin{tabular}{|lcc|}
$\begin{array}{l}\text { Duration of PPROM in } \\
\text { hours }\end{array}$ & No. of cases & Percentage \\
\hline$<12$ & 2 & $5.71 \%$ \\
\hline $13-24$ & 6 & $17.14 \%$ \\
\hline$>24$ & 27 & $77.14 \%$ \\
\hline
\end{tabular}

But no maternal mortality was seen in this study. In this study maternal morbidity was $35 \%$. There are various causes of maternal morbidity including fever, wound infection, lower respiratory tract infection (LRTI) retained placenta which required manual removal of placenta (MROP) and post-partum hemorrhage $(\mathrm{PPH})$.

Table 2: Maternal morbidity causes

\begin{tabular}{lcc} 
Morbidity & No. & \% \\
Puerperal pyrexia & 7 & 20 \\
\hline Chorioamnionitis & 7 & 20 \\
PPH & 6 & 17 \\
\hline UTI & 7 & 20 \\
\hline Wound infection & 6 & 17 \\
Sepsis & 2 & 6
\end{tabular}

Out of this, in this study Puerperal pyrexia, Chorioamnonitis, UTI, each contributes 20\% causes of maternal morbidity.

Table 3: Mode of delivery and parity

\begin{tabular}{lccccccc}
$\begin{array}{l}\text { Mode } \\
\text { of } \\
\text { delivery }\end{array}$ & $\begin{array}{c}\text { No } \\
\text { of } \\
\text { cases }\end{array}$ & $\begin{array}{c}34 \\
\text { wks }\end{array}$ & $\begin{array}{c}\text { \% } \\
\text { wks }\end{array}$ & & & $\begin{array}{c}36 \\
\text { wks }\end{array}$ & $\%$ \\
\hline NVD & 42 & 12 & 28.5 & 11 & 26.1 & 19 & 45.2 \\
LSCS & 58 & 23 & 39.6 & 16 & 27.5 & 19 & 32.7
\end{tabular}

In above table mode of delivery is categorized according to parity. The total number of cases of LSCS in this study was $58 \%$ in comparison to NVD $42 \%$.

In this study shows normal vaginal delivery percentage increases as gestational age increases from 34 to 36 weeks.
Table 4: Indications for LSCS in PPROM

\begin{tabular}{|lcc|}
\hline Indications & No. of cases & Percentage \\
\hline Fetal distress & 26 & $44.9 \%$ \\
\hline Non Progress Of Labour & 21 & $36.2 \%$ \\
\hline Mal position & 6 & $10.3 \%$ \\
\hline CPD & 5 & $8.6 \%$ \\
Total & 58 & $100 \%$
\end{tabular}

In this study LSCS was done in $58 \%$ of the cases, the main indications being fetal distress $44.9 \%$ followed by non progress of labour in $36.2 \%$, malposition in $10.3 \%$, CPD in $8.6 \%$.

Table 5: Perinatal morbidity

\begin{tabular}{|c|c|c|}
\hline Causes & No. of cases & Percentage \\
\hline Hyperbilirubinemia & 22 & $31.88 \%$ \\
\hline Sepsis & 15 & $21.73 \%$ \\
\hline Birth Asphyxia & 12 & $17.39 \%$ \\
\hline Conjunctivitis & 07 & $10.14 \%$ \\
\hline RDS & 07 & $10.14 \%$ \\
\hline Skin Infection & 02 & $2.89 \%$ \\
\hline Hyaline Membrane Disease & 01 & $1.44 \%$ \\
\hline Neonatal Meningitis & 03 & $4.34 \%$ \\
\hline
\end{tabular}

In this study perinatal morbidity was $69 \%$. There are various causes including hyperbilirubinemia (31.88\%), sepsis (21.7\%), Birth Asphyxia $(17.39 \%)$.

Other causes being conjunctivitis, Respiratory distress syndrome (RDS), Neonatal meningitis, skin infection, Hyaline membrane disease.

Only one $(1 \%)$ baby died because of very low birth weight

Table 6: Perinatal morbidity and mortality in relation to duration of PPROM

\begin{tabular}{|lccc|}
$\begin{array}{l}\text { Duration } \\
\text { Prom }\end{array}$ & $\begin{array}{c}\text { No of } \\
\text { cases }\end{array}$ & $\begin{array}{c}\text { Perinatal } \\
\text { morbidity }\end{array}$ & Percentage \\
\hline$<12 \mathrm{hrs}$ & 29 & 3 & 10.34 \\
\hline $12-24 \mathrm{hrs}$ & 19 & 9 & 36.84 \\
\hline $24-36 \mathrm{hrs}$ & 24 & 17 & 25 \\
\hline$>36 \mathrm{hrs}$ & 28 & 23 & 60.71 \\
\hline
\end{tabular}

Table 7: Perinatal morbidity according to birth weight

\begin{tabular}{|l|cc|c|}
\hline Birth weight & $\begin{array}{c}\text { No. of } \\
\text { cases }\end{array}$ & $\begin{array}{c}\text { Perinatal } \\
\text { morbidity }\end{array}$ & Percentage \\
\hline$<1800 \mathrm{~g}$ & 24 & 23 & $95.8 \%$ \\
\hline $1801-2000 \mathrm{~g}$ & 10 & 08 & $80 \%$ \\
\hline $2001-2200 \mathrm{~g}$ & 12 & 11 & $91.6 \%$ \\
\hline $2201-2500 \mathrm{~g}$ & 34 & 19 & $55.8 \%$ \\
\hline$>2501 \mathrm{~g}$ & 20 & 08 & $40 \%$ \\
\hline
\end{tabular}




\section{Discussion}

Preterm PROM complicates 3-8\% of pregnancies and leads to one third of preterm deliveries. ${ }^{2}$ It increases the risk of prematurity and leads to other perinatal and neonatal complications with 1-2\% risk of fetal death. It is an observational study done in tertiary care hospital including 100 patients of preterm premature rupture of membranes in between 34-37 weeks gestation admitted in labor room for a period of one year were studied.

\section{Maternal age}

In this study PPROM was present in $84 \%$ of cases in the age group of 21-30 years.

\section{Socio-economic status}

In this study the patients of low socioeconomic status were $67 \%$ and middle socioeconomic status were $28 \%$ which is comparable with the study by Shehla which is $68.23 \%$ and $31.77 \%$ respectively. ${ }^{4}$ Studies have shown that defects in the amniotic membranes occur due to low socioeconomic status associated with factors like malnutrition, over exertion, poor hygiene, stress, high parity, recurrent genitourinary infection and anemia. The risk of PPROM increases with decrease antibacterial activity in the amniotic fluid of patients with low socio- economic status.

\section{Booked and unbooked cases}

In this study the booked cases were $33 \%$ and unbooked cases $67 \%$. In unbooked cases there is lack of antenatal care leading to lack of identification of recurrent risk factors like PPROM, preterm delivery, induced abortions and their managements. Also, urogenital infections are not detected and treated due to lack of antenatal care leading to PPROM.

\section{Parity}

Primigravida is a risk factor for PPROM due to increased sexual activity \& increased sexual infection.
Table 8: Comparison of parity with other studies

\begin{tabular}{l|c|c|ccc|}
$\begin{array}{c}\text { Other } \\
\text { studies }\end{array}$ & \multicolumn{2}{|c}{ Swathi $^{\mathbf{3}}$ Shehla $^{\mathbf{4}}$} & Okeye $^{\mathbf{5}}$ & Trinity $^{\mathbf{6}}$ & $\begin{array}{c}\text { Our } \\
\text { sstudy }\end{array}$ \\
\hline Primi & $48 \%$ & $44.7 \%$ & $29.1 \%$ & $55.9 \%$ & $61 \%$ \\
\hline Multi & $52 \%$ & $55.3 \%$ & $69.9 \%$ & $44.1 \%$ & $39 \%$ \\
\hline
\end{tabular}

\section{Mode of delivery}

In our study normal delivery were $42 \%$. LSCS were more $(58 \%)$ because as this is a tertiary hospital with good NICU set up most of the cases reffered from outside hospital for fetal condition.

With slight comporomise of fetus as shown in USG (oligohydraminous) or nonreactive CTG early termination done. With increase in maternal leucocyte cont, CRP levels or increase in maternal temperature termination of pregnancy done by LSCS.

Table 9: Admission Delivery Interval

$\begin{array}{cccc}\begin{array}{c}\text { Admission delivery } \\ \text { interval }\end{array} & <12 \mathrm{hrs} & 12-24 \mathrm{hrs} & >24 \mathrm{hrs} \\ \text { No of cases } & 60 & 26 & 14\end{array}$

In our study maximum cases (86\%) delivered within 24hours of admission to the Hospital.

According to Russels, $196280 \%$ established labour within 24hours.

Table 10: Comparison of mode of deliveries with other studies

\begin{tabular}{|c|c|c|c|}
\hline $\begin{array}{l}\text { Mode of } \\
\text { delivery }\end{array}$ & NVD & SCS & INSRTUMENTAL \\
\hline Shehla ${ }^{4}$ & $65.88 \%$ & $14.11 \%$ & $20 \%$ \\
\hline Trinity $^{6}$ & $71.4 \%$ & $26.7 \%$ & $1.9 \%$ \\
\hline Kadikar $^{7}$ & $77 \%$ & $10 \%$ & $4 \%$ \\
\hline Chales PJ & $34.3 \%$ & $58.7 \%$ & $7 \%$ \\
\hline Our study & $42 \%$ & $58 \%$ & $0 \%$ \\
\hline
\end{tabular}

Table 11: Comparison of maternal morbidity with other studies

\begin{tabular}{lc} 
Other Studies $^{3}$ & Maternal Morbidity \\
Swathi $^{3}$ & $9 \%$ \\
Okeye $^{5}$ & $20 \%$ \\
Kadikar $^{7}$ & $8 \%$ \\
Anjana $^{12}$ & $21 \%$ \\
Our study & $35 \%$ \\
\hline
\end{tabular}

\section{Indication for LSCS}

Indications for LSCS In this study LSCS was done in $58 \%$ of the cases, the main indications 
being fetal distress $44.9 \%$ followed by non progress of labour in $36.2 \%$, malposition in $10.3 \%$, CPD in $8.6 \%$.

\section{Investigations for evidence of infection}

The investigations like total count, C-reactive protein and high vaginal swab for culture and sensitivity were done to evaluate for the evidence of infection. Leukocytosis can be affected by pregnancy and labor. CRP estimates seem to be reliable monitoring tool (Carroll). ${ }^{8}$ But in more detailed studies WBC and CRP were poor predictors of the presence of a positive amniotic fluid or fetal blood culture. 10

In this study 59 cases of normal vaginal flora, 14 cases of E. coli, 9 cases of Group B Streptococcus, 8 cases of Klebsiella, 4 cases of Neisseria gonorrhea , 3 cases of staphylococcus Aureus, 3 cases of Chlamydia trachomatis were isolated.

\section{AFI $<5$ and LSCS}

The findings of this study correlate with the studies by Tavassoli et al that PPROM with oligohydramnios is associated with shorter latency, higher rate of $\mathrm{C} / \mathrm{S}$, higher rate of neonatal morbidity and lower neonatal Apgar. ${ }^{11}$ Therefore, it is recommended to consider the AFI as a prognosis index in patients with PPROM. These patients with reduced AFI on NST had variable deceleration. These studies suggest that NST could be used to monitor for low AFI and cord compression in patients with PPROM. As the duration of PPROM increases the maternal morbidity also increases. The maternal morbidity in this study was 35\%. In this study $65 \%$ ofpatients were healthy. Febrile morbidity was seen in $07 \%$ of cases, $6 \%$ of cases had Post partum hemorrhage.

The study conducted by Arul Kumar showed that after 32 weeks of gestation the common causes of perinatal morbidity were RDS, perinatal asphyxia and infection, but with good supportive neonatal care most of the infants can survive. ${ }^{13}$ In this study perinatal morbidity was $69 \%$ of which $22 \%$ were hyperbilirubinemia $15 \%$ sepsis and $12 \%$ birth asphyxia. In this study, perinatal mortality was $1 \%$. The high incidence of maternal and neonatal infection may be consequence of decreased antibacterial activity in the amniotic fluid which is low in early pregnancy and increases with gestational age. Another factor is the limited ability of a preterm infant to fight infection.

\section{Perinatal morbidity and mortality in relation to duration of PPROM}

In this study, as the duration of PPROM increases, perinatal morbidity increases. When PPROM to delivery interval more than 36 hours Perinatal morbidity was $60.71 \%$. The studies by Russel showed that the danger of infection to both mother and fetus increases with duration of PPROM. But prolongation of latent period decreases the incidence of RDS. 14

\section{Perinatal morbidity and mortality according to birth weight}

Perinatal morbidity \& mortality decreases as the birth weight increases. When the weight was $<1800$ grams perinatal morbidity was $95.8 \%$. It reduced to $40 \%$ morbidity when birth weight increases to $>2500$ grams.

\section{Conclusion}

PPROM is one of the important causes of preterm birth that can result in high perinatal morbidity and mortality along with maternal morbidity. Looking after a premature infant puts immense burden on the economy and health care resources of the country. Therefore, management of PPROM requires accurate diagnosis and evaluation of the risks and benefits of continued pregnancy or expeditious delivery. An understanding of gestational age dependent neonatal morbidity and mortality is important in determining the potential benefits of conservative management of preterm PROM at any gestation. It is important that the patient should be well informed regarding the potential for subsequent 
maternal, fetal and neonatal complications regardless of the management approach. Risk scoring strategies involving the demographic variable along with previous history of preterm deliveries should be developed to identify high risk cases and treating them prior to rupture of membrane. Tocolysis in women with PPROM is needed till completion of corticosteroid. Antenatal corticosteroids should be administered in women with PPROM. Routine antibiotic administration reduces maternal and neonatal morbidity. Antibiotic therapy also delays delivery, thereby allowing sufficient time for prophylactic prenatal corticosteroids to take effect. PPROM with oligohydramnios is associated with shorter latency, non reactive $\mathrm{CTG}$, higher rate of cesarean sections,

\section{Recommendations}

- Regular antenatal care, good hygiene, nutritious diet, early diagnosis of vaginal infection, literacy, and health education can decrease the incidence of PROM.

- Timely referral of PROM cases to tertiary care hospitals and timely intervention can further improve perinatal outcome.

- Strict aseptic precautions, appropriate therapy, regular antenatal follow-up are important factors in the prevention and management of PPROM.

- The management protocol should be improved and strictly followed in order to improve neonatal outcomes.

- Thus, PPROM patient should be considered high risk and monitored closely with strict supervision and managed according to protocol.

\section{References}

1. Caughey AB, Robinson JN, Norwitz ER. Contemporary Diagnosis and Management of Preterm Premature Rupture of Membranes. Rev. Obstet Gynecol. 2008;1(1):11-22.

2. Fernando Arias; Premature rupture of the membrane, "Practical guide to high risk pregnancy and delivery". $3^{\mathrm{rd}} \mathrm{Ed}$; Elsevier, New Delhi, 2008;220-237.

3. Pandey S, Dave A, Bandi S. Maternal and fetal outcome in cases of preterm premature rupture of membranes. J ObstetGynaecol India.2000;50:63.

4. Noor S, Fawwad A, Shahzad H, Sultana R, Bashir R. Foetomaternal outcome in patients with or without premature rupture of membranes. J Ayub Medical College Abbottabad.2010;22(1):164-7.

5. Okeke TC, Enwereji JO, Okoro OS, Adiri CO, Ezugwu EC, Agu PU, et al. The incidence and management outcome of preterm premature rupture of membranes in a tertiary hospital in Nigeria. Am J Clinic Med Res. 2014;(2)1:14-17.

6. Triniti A, Suthatvorawut S, Pratak O. Epidemiologic study of cervical swab culture in preterm premature rupture of membrane (PPROM) at Ramathibodi Hospital. Thai J ObstetGynaecol.2008;16(3):173-8.

7. Kadikar GK, Gandhi MR, Damani SK. A Study of Feto-Maternal Outcome in Cases of Premature Rupture of Membrane. IJSR. 2014;3(3):299-301

8. Carroll SG, Philpott-H J, Nicolaides KH. Amniotic fluid gram stain and leukocyte count in the prediction of intrauterine infection in preterm prelabouramniorrhexis. Fetal Diag Therapy.1996;11(1):1-5.

9. Kamala Jayaram, ScailaSudha, A study of premature rupture of membranes Management and outcome. J ObstetGynaecol India.2001;51(2):58-60.

10. ACOG Committee on Practice Bulletins Obstetrics, authors. Clinical Management guidelines for Obstetrician-Gynecologists. (ACOG Practice Bulletin No 80: premature rupture of membranes). Obstet Gynecol. 2007;109(4):1007-19.

11. TavassoliF, Ghasemi M, Mohamadzade A, Sharifian J. Survey of pregnancy outcome in 
preterm premature rupture of membrane in AFI $<5$ and $>5$. Oman Med J. 2010;25(2):118-23.

12. Anjana D, Reddi R. Premature rupture of membrane: A clinical study. J ObstGynecol India1996;46:63-76.

13. Kumaran A, Penna LK. prelabor ROM. Management of labor, Orient Longman.2005:306-18.

14. Russell KP, Anderson GV. The aggressive management of ruptured membranes. Am J Obstet Gynecol. 1962;83(7):930-7. 